\section{Gold Bulletin Papers: The ‘Top 10’ for April!}

The following is a list of the most frequently downloaded papers contained in the Gold Bulletin archive at www.goldbulletin.org for the month of April 2005:

118 Carat Yellow Gold Alloys With Increased Hardness

Rainer Süss, Elma van der Lingen and Lizelle Glaner, 37, No. 3, 2004

2 The Chemistry of Gold-Fluoro Compounds - a continuing challenge for gold chemists

Fabian Mohr, 37, No 3, 2004

3 Gold as a Novel Catalyst in the 21st Century: Preparation, Working Mechanisms and Applications

Masatake Haruta, 37, No. 1, 2004

4 Homogeneous Catalysis by Gold

Stephen Hashmi, 37, No. 1, 2004

5 Catalytic Gold

Johannes Schwank, 16, No. 4, 1988

6 Photocatalysis by Au Nanoparticles: Reforming of Methanol

Michael Bowker, 37, No. 3, 2004

7 The Weird World of the Nanoscale Gold

Michael Cortie, 37, No. 3, 2004

8 Surface Chemistry of Catalysis by Gold

R Meyer, C Lemire, Sh K Shaikhutdinov and Hans-Joachim Freund, 37, No.1, 2004

9 Electrocatalytic Reduction of Oxygen: Gold and GoldPlatinum Nanoparticle Catalysts Prepared by Two Phase Protocol

Mathew M Maye, Nancy N Kariuki, Jin Luo, Li Han, Peter Njoki, Lingyan Wang, Yan Lin, $\mathrm{H}$ Richard Naslund and Chuan-Jian Zhong, 37, No. 3, 2004

10 Identification of Active Sites and Influence of Real Structure of Gold Catalysts in the Selective Hydrogenation of Acrolein to Allyl alcohol

Peter Claus, 37, No. 3, 2004
In March, the Top 10 was different although, not surprisingly, there is much overlap:

1 Suss et al, 37(3), 2004

2 Cortie, 37(1), 2004

3 Meyer et al, 37(1), 2004

4 Mohr, 37(3), 2004

5 Haruta, 37(1), 2004

6 Bowker, 37(3), 2004

7 Bond \& Thompson, 33(2), 2000

8 Volkov, 37(3), 2004

9 Hashmi, 37(1),2004

10 Dong, 37(3), 2004

If we look at the top 20 in March, 'old' papers by Schmidbaur [23(1), 1990] and Hutchings [29(4), 1996] featured, indicating their importance, along with the BondThompson paper, to the fields of chemistry and catalysis respectively as core papers reviewing the current status of science. The strong showing of catalyst papers for both months reflects the strong current high interest in this topic.

Other items featuring very strongly are the 'Gold in the News' and the Highlights of literature and patents abstracts. 